\title{
Efficient Solutions to Bargaining Problems with Uncertain Disagreement Points
}

Citation for published version (APA):

Bossert, W., \& Peters, H. J. M. (2002). Efficient Solutions to Bargaining Problems with Uncertain Disagreement Points. Social Choice and Welfare, 19, 489-502. https://doi.org/10.1007/s003550100124

Document status and date:

Published: 01/01/2002

DOI:

10.1007/s003550100124

Document Version:

Publisher's PDF, also known as Version of record

\section{Please check the document version of this publication:}

- A submitted manuscript is the version of the article upon submission and before peer-review. There can be important differences between the submitted version and the official published version of record.

People interested in the research are advised to contact the author for the final version of the publication, or visit the DOI to the publisher's website.

- The final author version and the galley proof are versions of the publication after peer review.

- The final published version features the final layout of the paper including the volume, issue and page numbers.

Link to publication

\footnotetext{
General rights rights.

- You may freely distribute the URL identifying the publication in the public portal. please follow below link for the End User Agreement:

www.umlib.nl/taverne-license

Take down policy

If you believe that this document breaches copyright please contact us at:

repository@maastrichtuniversity.nl

providing details and we will investigate your claim.
}

Copyright and moral rights for the publications made accessible in the public portal are retained by the authors and/or other copyright owners and it is a condition of accessing publications that users recognise and abide by the legal requirements associated with these

- Users may download and print one copy of any publication from the public portal for the purpose of private study or research.

- You may not further distribute the material or use it for any profit-making activity or commercial gain

If the publication is distributed under the terms of Article $25 \mathrm{fa}$ of the Dutch Copyright Act, indicated by the "Taverne" license above, 


\title{
Efficient solutions to bargaining problems with uncertain disagreement points
}

\author{
Walter Bossert ${ }^{1}$, Hans Peters ${ }^{2}$ \\ 1 Département de Sciences Economiques and C.R.D.E., Université de Montréal, \\ C.P. 6128, succursale Centre-ville, Montréal QC H3C 3J7, Canada \\ (e-mail: Walter.Bossert@umontreal.ca) \\ 2 Department of Quantitative Economics, University of Maastricht, P.O. Box 616, \\ 6200 MD Maastricht, The Netherlands (e-mail: h.peters@ke.unimaas.nl)
}

Received: 17 March 2000/Accepted: 15 January 2001

\begin{abstract}
We consider a cooperative model of bargaining where the location of the disagreement point may be uncertain. Based on the maximin criterion, we formulate an ex ante efficiency condition and characterize the class of bargaining solutions satisfying this axiom. These solutions are generalizations of the monotone path solutions. Adding individual rationality yields a subclass of these solutions. By employing maximin efficiency and an invariance property that implies individual rationality, a new axiomatization of the monotone path solutions is obtained. Furthermore, we examine the consequences of employing efficiency axioms based on alternative decision criteria.
\end{abstract}

\section{Introduction}

The purpose of the cooperative model of bargaining as formulated by Nash (1950) is to recommend outcomes for bargaining situations involving two or more agents. A bargaining solution assigns a chosen utility vector to each bargaining problem represented by the corresponding set of feasible utility vectors and the disagreement point - the utility vector that results if the agents fail to reach an agreement. Our interpretation of the bargaining problem is normative. We think of the chosen utility vector as a recommended outcome of a bargaining situation (for example, the outcome suggested by an impartial arbitrator).

Financial support from the Nederlandse Organisatie voor Wetenschappelijk Onderzoek (NWO) under grant no. B46-363 and from the University of Nottingham is gratefully acknowledged. We thank an associate editor and two referees for comments. 
Whereas most of the early contributions to the theory of bargaining (such as, for example, Nash 1950; Kalai and Smorodinsky 1975; Kalai 1977) focus on properties of bargaining solutions with respect to changes in the feasible set, there is now a substantial literature dealing with the role of the disagreement point in establishing a solution outcome. For example, Thomson (1987), Wakker (1987), Livne (1989), and Bossert (1994) analyze monotonicity properties of bargaining solutions with respect to changes in the disagreement point.

Properties of bargaining solutions that are motivated by the presence of uncertainty regarding the location of the disagreement point are examined, for instance, in Livne (1988), Chun (1989), Chun and Thomson (1990a,b,c), and Peters and van Damme (1991). These contributions analyze the consequences of imposing axioms such as disagreement point concavity and related conditions. Disagreement point concavity requires that agents weakly prefer to solve a contingent problem with an uncertain disagreement point immediately on the basis of their expected payoffs rather than waiting until the uncertainty is resolved. See, for example, Chun and Thomson (1990a,b) for details and Thomson (1994) for a survey and further references.

In this paper, we address the uncertainty issue with respect to the disagreement point from another angle. We define bargaining problems under uncertainty by specifying the disagreement points that could materialize in different states of the world with a fixed feasible set of utility vectors. We then impose an efficiency condition based on the maximin criterion and show that this axiom is satisfied only by a specific class of solutions that are generalizations of the monotone path solutions (see, for example, Thomson and Myerson 1980). Furthermore, we illustrate how this class of solutions can be narrowed down by imposing individual rationality in addition to efficiency under uncertainty. Together with a strengthening of individual rationality, maximin efficiency is used to provide a characterization of the class of monotone path solutions.

According to Wald (1950, Sect. 1.4.2), the use of the maximin criterion seems reasonable "when an a priori distribution [...] does not exist or is unknown [...]'. See also Gilboa and Schmeidler (1989), who formalize Wald's remark by appealing to a notion of uncertainty aversion. Alternatively, the use of the maximin criterion can be justified by appealing to more general setbased models of choice under uncertainty. In those approaches, the only information available to a decision maker is the set of possible outcomes that could materialize. See, for example, Pattanaik and Peleg (1984) for a detailed discussion and justification of this approach. Once this approach is adopted, imposing some weak properties on a ranking of sets of possible outcomes implies that the ranking must be based on best and worst elements only. For example, Kannai and Peleg (1984), Barberà et al. (1984), and Bossert et al. (2000) present results of that nature; see also Arrow and Hurwicz (1972) for this implication in a more traditional decision-theoretic context. Within the class of those rankings, maximin (the ranking paying attention to the worst outcome only) plays an important role because it is associated with a notion 
of uncertainty aversion (see Bossert 1997, for a definition and discussion of uncertainty aversion in set-based models of choice under uncertainty).

The approach in this paper complements the one followed in Bossert et al. (1996) and in Bossert and Peters (2001), where efficiency conditions with respect to various decision criteria are examined in bargaining models with uncertain feasible sets. Despite the conceptual similarity between those two approaches, some of the techniques employed here are quite different from those used in the above-mentioned earlier contributions, due to the restricted flexibility that obtains when feasible sets are assumed to be fixed. Because variations in the disagreement point appear to be easier to simulate in an experimental setting than (possibly very complex) changes in the feasible set of utility vectors, the model developed in this paper provides an interesting framework for empirical investigations in the analysis of bargaining situations. This is, of course, a feature shared by other contributions that focus on the effects of variations in the disagreement point with fixed feasible sets.

Section 2 introduces our basic notation and definitions. Efficient bargaining with respect to the maximin criterion for problems with uncertain disagreement points is analyzed in Sect. 3. To conclude the paper, a discussion of alternative decision criteria is provided in Sect. 4.

\section{Preliminaries}

Let $\mathbb{R}\left(\mathbb{R}_{+}, \mathbb{R}_{++}, \mathbb{R}_{--}\right)$denote the set of real numbers (nonnegative, positive, negative real numbers). For a positive integer $n \geq 2, \mathbb{R}^{n}\left(\mathbb{R}_{+}^{n}, \mathbb{R}_{++}^{n}, \mathbb{R}_{--}^{n}\right)$ is the $n$-fold Cartesian product of $\mathbb{R}\left(\mathbb{R}_{+}, \mathbb{R}_{++}, \mathbb{R}_{--}\right)$. The origin of $\mathbb{R}^{n}$ is denoted by $\mathbf{0}$. The inner product of two vectors $x, y \in \mathbb{R}^{n}$ is denoted by $x \cdot y$. Our notation for vector inequalities is as follows. For all $x, y \in \mathbb{R}^{n}, x \geq y$ if $x_{i} \geq y_{i}$ for all $i=1, \ldots, n$, and $x>y$ if $x_{i}>y_{i}$ for all $i=1, \ldots, n$. A set $S \subset \mathbb{R}^{n}$ is comprehensive if, for all $x \in S$ and all $y \in \mathbb{R}^{n}$ such that $x \geq y$ and $x \neq y$, we have $y \in S$; and strictly comprehensive if, additionally, there exists $z \in S$ such that $z>y$. The set $S$ is bounded from above if there exist $p \in \mathbb{R}_{++}^{n}$ and $\alpha \in \mathbb{R}$ such that $p \cdot x \leq \alpha$ for every $x \in S$. The interior of $S \subset \mathbb{R}^{n}$ is denoted by $I(S)$. For $x \in \mathbb{R}^{n}$ and $S \subset \mathbb{R}^{n}$, we define $x+S=S+x=$ $\left\{y \in \mathbb{R}^{n} \mid \exists z \in S\right.$ such that $\left.y=x+z\right\}$.

Let $N=\{1, \ldots, n\}$ with $n \geq 2$ be the (fixed) set of agents involved in a bargaining problem. A set $S \subset \mathbb{R}^{n}$ is called admissible if it is nonempty, closed, strictly comprehensive and bounded from above. By $\Sigma$ we denote the collection of all admissible sets. A pair $(S, d)$ with $S \in \Sigma$ and $d \in I(S)$ is called a bargaining problem. Observe that $I(S)$ is nonempty by definition of $\Sigma$. $S$ represents the set of feasible utility vectors and $d$, the disagreement point, the utility vector that results if the agents in $N$ fail to reach an agreement. That $d$ is an interior point of $S$ is a standard assumption in bargaining theory to ensure that there are potential gains for all agents involved. For the current paper, this assumption is not crucial but we follow the standard convention and exclude bargaining problems where $d$ is on the boundary of $S$. The collection of all bargaining problems is denoted by $\mathscr{B}$. 
A (bargaining) solution is a mapping $F: \mathscr{B} \rightarrow \mathbb{R}^{n}$ such that $F(S, d) \in S$ for all $(S, d) \in \mathscr{B}$. We use strict comprehensiveness instead of the weaker comprehensiveness assumption in order to simplify the exposition. If comprehensive but not necessarily strictly comprehensive problems are permitted, additional regularity assumptions such as continuity are required. Furthermore, if strict comprehensiveness is weakened to comprehensiveness, our efficiency axioms should be modified as well in order to continue to be in line with the strong version of Pareto optimality used here. Because this alternative formulation increases the complexity of the exposition without leading to new insights, we have chosen to impose strict comprehensiveness. Note that we do not impose convexity of the feasible set $S$. The convexity assumption is often motivated by appealing to expected utility considerations. Because we investigate alternative decision criteria with respect to uncertainty in the disagreement point, it seems appropriate not to invoke any expected utility notions in defining the domain of a solution. It should be mentioned, however, that all of our results remain true if convexity is added in the definition of an admissible set and, therefore, the validity of our results does not rely on the availability of nonconvex problems.

Pareto optimality is a standard restriction imposed on bargaining solutions. This axiom requires that $F$ should not select a utility vector in $S$ that is dominated in the sense that all agents in $N$ could be made better off by moving to another point in $S$. In order to define this condition formally, let $P(S)=\{x \in S \mid \nexists y \in S \backslash\{x\}$ such that $y \geq x\}$ for all $S \in \Sigma$. Note that, due to the strict comprehensiveness assumption, the strong and the weak Pareto sets coincide.

Pareto optimality. For all $(S, d) \in \mathscr{B}, F(S, d) \in P(S)$.

Another standard axiom is individual rationality. It requires that none of the agents is worse off at a solution outcome than at the disagreement outcome. The set of individually rational points for $(S, d) \in \mathscr{B}$ is given by $\operatorname{IR}(S, d):=\{x \in S \mid x \geq d\}$.

Individual rationality. For all $(S, d) \in \mathscr{B}, F(S, d) \in I R(S, d)$.

A property that is of importance in this paper is a domination axiom. It requires that, for a given feasible set $S$ and two disagreement points $d$ and $d^{\prime}$, there is a domination relationship between the agents' gains $F(S, d)-d$ and $F\left(S, d^{\prime}\right)-d^{\prime}$. For any two problems with the same feasible set of utility vectors $S$, all agents' gains over the disagreement outcome in the solution outcome corresponding to the disagreement point $d$ are greater than or equal to the corresponding gains in the solution outcome obtained for the disagreement point $d^{\prime}$, or vice versa. This axiom turns out to be implied by our efficiency requirement for bargaining problems with uncertain disagreement points.

Domination. For all $S \in \Sigma$, for all $d, d^{\prime} \in I(S)$,

$$
F(S, d)-d \geq F\left(S, d^{\prime}\right)-d^{\prime} \quad \text { or } \quad F\left(S, d^{\prime}\right)-d^{\prime} \geq F(S, d)-d .
$$


The efficiency axiom that is of most importance in this paper is the ex ante efficiency property induced by the maximin criterion. In the larger part of this paper we study the consequences of this condition for the solution $F$. The relevance of this axiom is in its interpretation in terms of the agents' assessment of uncertain bargaining outcomes. Suppose that the feasible set $S$ is known but the disagreement point is uncertain: all that is known to the agents is that the disagreement point is one of the two vectors $d$ and $d^{\prime}$. Consequenctly, if the solution $F$ is to be employed, agent $i \in N$ 's gain will be $F_{i}(S, d)-d_{i}$ or $F_{i}\left(S, d^{\prime}\right)-d_{i}^{\prime}$, and the above criterion assumes that this agent (pessimistically) evaluates this uncertain gain by taking the minimum of these two numbers. In that case, ex ante efficiency of $F$ in the presence of this disagreement point uncertainty means that $F$ satisfies maximin efficiency.

In order to define this efficiency axiom formally, we use the following terminology. For $S \in \Sigma, d, d^{\prime} \in I(S)$, and $x, x^{\prime}, y, y^{\prime} \in S$, we say that the pair $\left(x, x^{\prime}\right)$ min-dominates the pair $\left(y, y^{\prime}\right)$ if

$$
\min \left\{x_{i}-d_{i}, x_{i}^{\prime}-d_{i}^{\prime}\right\}>\min \left\{y_{i}-d_{i}, y_{i}^{\prime}-d_{i}^{\prime}\right\}
$$

for all $i \in N$.

Maximin efficiency. For all $S \in \Sigma$, for all $d, d^{\prime} \in I(S)$, there exist no $x, x^{\prime} \in S$ such that $\left(x, x^{\prime}\right)$ min-dominates $\left(F(S, d), F\left(S, d^{\prime}\right)\right)$.

Maximin efficiency could be reformulated by considering situations with an arbitrary number of possible states of the world rather than restricting attention to two possible states. This alternative axiom is equivalent to the above version which we use in this paper to facilitate the exposition.

The optimistic counterpart of maximin efficiency is maximax efficiency. In that case, an agent uses the highest possible utility to evaluate contingent bargaining outcomes under disagreement point uncertainty. Analogously to mindomination, max-domination is defined as follows. For $S \in \Sigma, d, d^{\prime} \in I(S)$, and $x, x^{\prime}, y, y^{\prime} \in S$, we say that the pair $\left(x, x^{\prime}\right)$ max-dominates the pair $\left(y, y^{\prime}\right)$ if

$$
\max \left\{x_{i}-d_{i}, x_{i}^{\prime}-d_{i}^{\prime}\right\}>\max \left\{y_{i}-d_{i}, y_{i}^{\prime}-d_{i}^{\prime}\right\}
$$

for all $i \in N$. The corresponding efficiency property is maximax efficiency.

Maximax efficiency. For all $S \in \Sigma$, for all $d, d^{\prime} \in I(S)$, there exist no $x, x^{\prime} \in S$ such that $\left(x, x^{\prime}\right)$ max-dominates $\left(F(S, d), F\left(S, d^{\prime}\right)\right)$.

The consequences of imposing this alternative decision criterion will be discussed briefly in Sect. 4.

\section{Maximin efficiency and monotone path solutions}

We begin our investigation of the consequences of maximin efficiency by showing that maximin efficiency is equivalent to the conjunction of Pareto optimality and domination. This observation is employed in the proof of the main characterization result in this section and is stated in the following lemma. 
Lemma 1. A solution $F$ is maximin efficient if, and only if, $F$ is Pareto optimal and satisfies domination.

Proof. Assume that $F$ is maximin efficient. We first show Pareto optimality and then domination.

Suppose, to the contrary, that $F$ is not Pareto optimal. Then there is an $(S, d) \in \mathscr{B}$ and an $x \in S$ with $x>F(S, d)$. Thus, obviously, the pair $(x, x)$ min-dominates $(F(S, d), F(S, d))$, and we have a violation of maximin efficiency.

Next suppose, again to the contrary, that $F$ does not satisfy domination. Then there exist $S \in \Sigma, d, d^{\prime} \in I(S)$, and $i, j \in N$ with

$$
F_{i}(S, d)-d_{i}>F_{i}\left(S, d^{\prime}\right)-d_{i}^{\prime} \quad \text { and } \quad F_{j}(S, d)-d_{j}<F_{j}\left(S, d^{\prime}\right)-d_{j}^{\prime} .
$$

Let $N_{d} \subset N$ be the subset of agents $k \in N$ for which $F_{k}(S, d)-d_{k}>$ $F_{k}\left(S, d^{\prime}\right)-d_{k}^{\prime}$ and let $N_{d^{\prime}} \subset N$ be the subset of agents $k \in N$ for which $F_{k}(S, d)-d_{k}<F_{k}\left(S, d^{\prime}\right)-d_{k}^{\prime}$. By definition, $i \in N_{d}$ and $j \in N_{d^{\prime}}$. By the strict comprehensiveness of $S$, we can find an $x \in S$ by perturbing $F(S, d)$ and an $x^{\prime} \in S$ by perturbing $F\left(S, d^{\prime}\right)$ such that the following inequalities are satisfied:

$$
\begin{aligned}
& \text { for all } k \in N_{d}, \quad F_{k}(S, d)-d_{k}>x_{k}-d_{k}>x_{k}^{\prime}-d_{k}^{\prime}>F_{k}\left(S, d^{\prime}\right)-d_{k}^{\prime} ; \\
& \text { for all } k \in N_{d^{\prime}}, \quad F_{k}\left(S, d^{\prime}\right)-d_{k}^{\prime}>x_{k}^{\prime}-d_{k}^{\prime}>x_{k}-d_{k}>F_{k}(S, d)-d_{k} ; \\
& \text { for all } k \in N \backslash\left(N_{d} \cup N_{d^{\prime}}\right), \quad x_{k}>F_{k}(S, d) \quad \text { and } \quad x_{k}^{\prime}>F_{k}\left(S, d^{\prime}\right) .
\end{aligned}
$$

Then, by construction, $\left(x, x^{\prime}\right)$ min-dominates $\left(F(S, d), F\left(S, d^{\prime}\right)\right)$, contradicting maximin efficiency.

Now assume that $F$ is Pareto optimal and satisfies domination. Suppose, contrary to what we want to prove, that $F$ does not satisfy maximin efficiency. Then there are $S \in \Sigma, d, d^{\prime} \in I(S)$, and $x, x^{\prime} \in S$ such that the pair $\left(x, x^{\prime}\right)$ mindominates the pair $\left(F(S, d), F\left(S, d^{\prime}\right)\right)$. By domination, without loss of generality, $F_{i}(S, d)-d_{i} \leq F_{i}\left(S, d^{\prime}\right)-d_{i}^{\prime}$ for all $i \in N$. Then, for all $i \in N$,

$$
\begin{aligned}
x_{i}-d_{i} & \geq \min \left\{x_{i}-d_{i}, x_{i}^{\prime}-d_{i}^{\prime}\right\}>\min \left\{F_{i}(S, d)-d_{i}, F_{i}\left(S, d^{\prime}\right)-d_{i}^{\prime}\right\} \\
& =F_{i}(S, d)-d_{i} .
\end{aligned}
$$

This implies $F(S, d) \notin P(S)$, a violation of Pareto optimality.

Now we turn to a characterization of the class of all solutions satisfying maximin efficiency. This class is a generalization of the class of monotone path solutions (see, for example, Thomson and Myerson 1980). In order to introduce those solutions formally, we need some further definitions.

A monotone path is a subset $X$ of $\mathbb{R}^{n} \backslash \mathbb{R}_{--}^{n}$ such that, for all $x, x^{\prime} \in X$, $x \geq x^{\prime}$ or $x^{\prime} \geq x$. We say that a monotone path $X$ is compatible with an admissible set $S \in \Sigma$ if, for all $d \in I(S),(d+X) \cap P(S) \neq \varnothing$. Observe that by the monotonicity property of a monotone path the latter set can contain at most one point.

The solution $F$ is a generalized monotone path solution if for every $S \in \Sigma$, there is a monotone path $\Phi^{S}$ compatible with $S$ such that, for every $(S, d) \in \mathscr{B}$, 
we have $\{F(S, d)\}=\left(d+\Phi^{S}\right) \cap P(S)$. In that case, we say that $F$ is generated by the collection $\left\{\Phi^{S} \mid S \in \Sigma\right\}$. In geometrical terms, given a monotone path $\Phi^{S}$ compatible with $S \in \Sigma$, the corresponding solution outcome for $(S, d) \in \mathscr{B}$ is obtained by translating $\Phi^{S}$ using the translation vector $d$ and intersecting the resulting set with the Pareto frontier of $S$. The class of generalized monotone path solutions - unlike the class of monotone path solutions discussed in the earlier literature - contains solutions that are not individually rational. $F$ is an individually rational generalized monotone path solution if $F$ is a generalized monotone path solution generated by a collection $\left\{\Phi^{S} \mid S \in \Sigma\right\}$ such that $\Phi^{S} \subset \mathbb{R}_{+}^{n}$ for all $S \in \Sigma$. Individual rationality is ensured if all monotone paths $\Phi^{S}$ are such that they do not contain any nonpositive points as in the definition of the individually rational generalized monotone path solutions. Finally, $F$ is a monotone path solution if $F$ is an individually rational generalized monotone path solution such that $\Phi^{S}=\Phi^{T}$ for all $S, T \in \Sigma$.

The reason why even solutions violating standard properties such as individual rationality are included in the class of generalized monotone path solutions is that our first objective is to identify all solutions satisfying maximin efficiency. We then characterize subclasses thereof by imposing further axioms. A graphical illustration of the construction of some generalized monotone path solutions is provided in Fig. 1 (the individually rational case) and in Fig. 2 (without individual rationality).

The compatibility requirement defined above is important to ensure that the solution generated by a collection of monotone paths is well-defined. A monotone path compatible with one admissible set does not have to be compatible with another feasible set. It is easy to verify that a monotone path compatible with an admissible set $S$ is connected and unbounded from above. However, it may or may not be bounded from below. For example, the monotone path $X=\left\{(t, t-1) \mid t \in \mathbb{R}_{++}\right\}$is compatible with all $S \in \Sigma$ and bounded from below, whereas the path $X^{\prime}=\left\{(t, \ln (t)) \mid t \in \mathbb{R}_{++}\right\}$is compatible with all admissible feasible sets as well but $X^{\prime}$ obviously is not bounded from below.

We now obtain the following characterization of the class of generalized monotone path solutions which, to the best of our knowledge, has not been discussed and axiomatized in the earlier literature.

Theorem 1. A solution $F$ is maximin efficient if, and only if, it is a generalized monotone path solution.

Proof. It is easy to see that a generalized monotone path solution is Pareto optimal and satisfies domination. Therefore the if-part of the theorem follows by Lemma 1.

For the only-if part assume that $F$ is maximin efficient. Hence, by Lemma $1, F$ is Pareto optimal and satisfies domination. Fix $S \in \Sigma$, and define

$$
\Phi^{S}:=\left\{x \in \mathbb{R}^{n} \mid x=F(S, d)-d \text { for some } d \in I(S)\right\} .
$$

By Pareto optimality, $x \notin \mathbb{R}_{--}^{n}$ for all $x \in \Phi^{S}$, and by domination, $x \leq x^{\prime}$ or $x \geq x^{\prime}$ for all $x, x^{\prime} \in \Phi^{S}$. So $\Phi^{S}$ is a monotone path. It follows directly that 


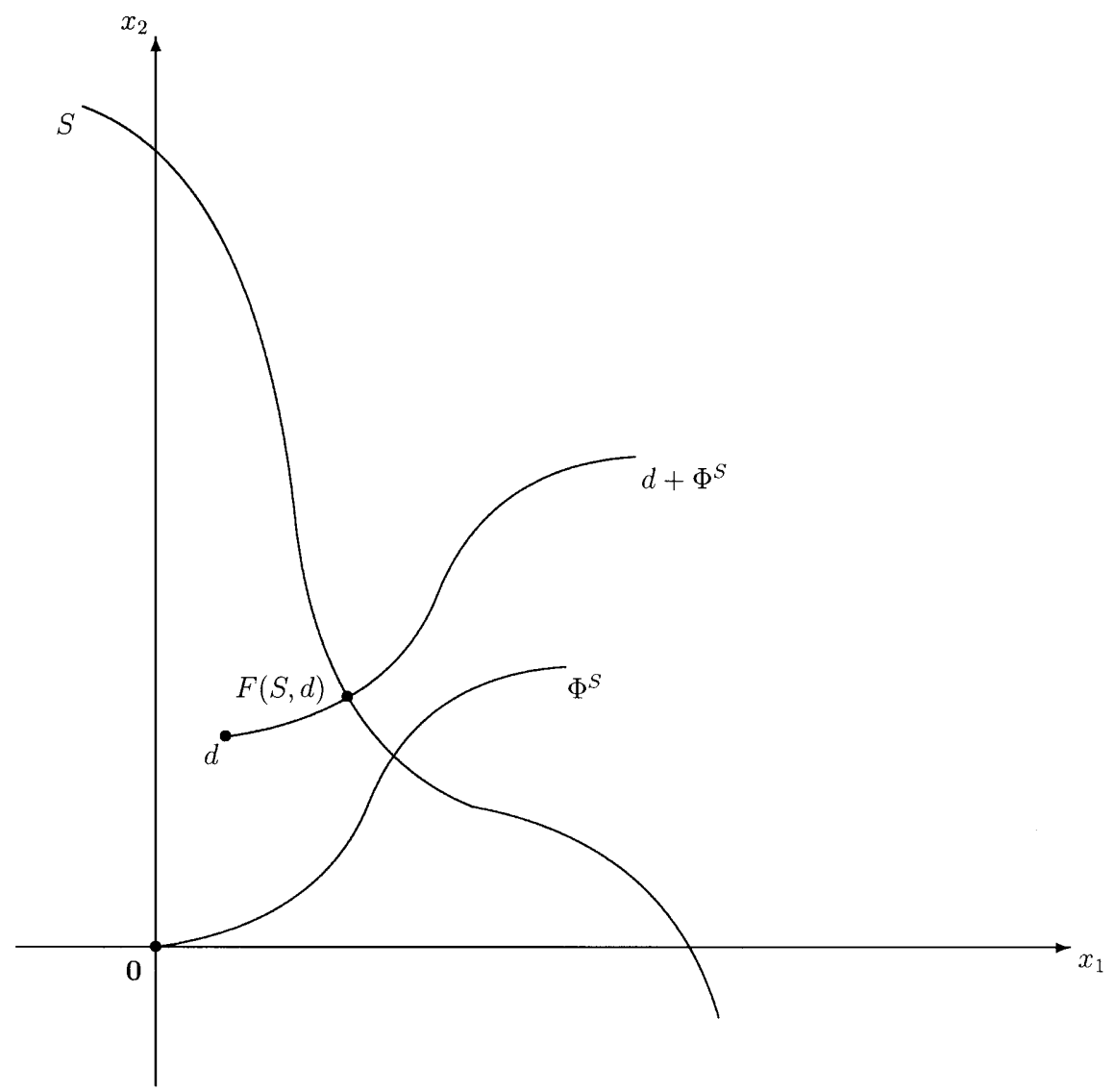

Fig. 1 An individually rational generalized monotone path solution

$\Phi^{S}$ is compatible with $S$ and that, in particular, $\{F(S, d)\}=\left(d+\Phi^{S}\right) \cap P(S)$ for every disagreement point $d \in I(S)$.

By adding individual rationality to maximin efficiency, a characterization of the individually rational generalized monotone path solutions is obtained. Note that this class is still considerably larger than the class of monotone path solutions themselves because no restrictions are imposed on the relationship between the monotone paths for different admissible sets. The proof of this characterization result is immediate and is therefore omitted.

Theorem 2. A solution $F$ is maximin efficient and individually rational if, and only if, it is an individually rational generalized monotone path solution.

We now add a stronger axiom than individual rationality to maximin efficiency in order to obtain a characterization of the monotone path solutions. This axiom requires that the agents' utility gains over the disagreement utility 


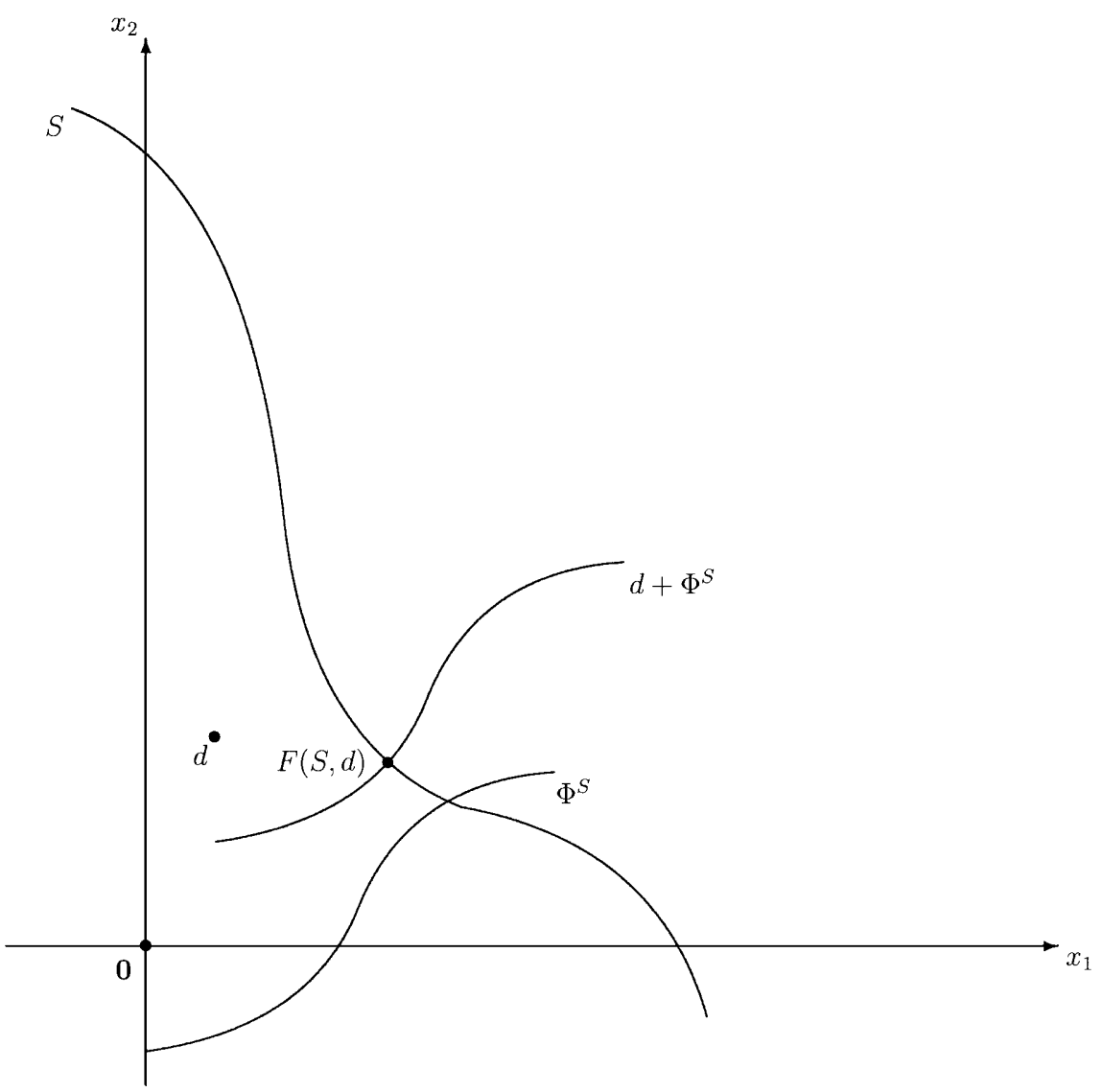

Fig. 2 A nonindividually rational generalized monotone path solution

in a solution outcome are invariant with respect to translations that leave the set of these gains in a problem unchanged.

$I R$-invariance. For all $(S, d),\left(T, d^{\prime}\right) \in \mathscr{B}$, if $\operatorname{IR}(S-d, \mathbf{0})=\operatorname{IR}\left(T-d^{\prime}, \mathbf{0}\right)$, then $F(S, d)-d=F\left(T, d^{\prime}\right)-d^{\prime}$.

IR-invariance combines a translation invariance property with an independence of nonindividually rational alternatives property. It only applies to situations where the individually rational portions of two feasible sets are translations of each other and, therefore, it is not subject to some of the criticisms raised against Nash's (1950) independence of irrelevant alternatives. See, for example, Peters (1992) for a discussion of axioms of that nature. Note that IR-invariance is implied by the conjunction of independence of nonindividually rational alternatives and translation invariance. Because the weaker axiom is sufficient to obtain our results, we use it instead of imposing independence of nonindividually rational alternatives and translation invariance. As a preliminary result, we state the following lemma. 
Lemma 2. If a solution $F$ is Pareto optimal and IR-invariant, then $F$ is individually rational.

Proof. Suppose, by way of contradiction, that $F$ satisfies Pareto optimality and $I R$-invariance but violates individual rationality. Then there exists $(S, d) \in \mathscr{B}$ such that $F(S, d) \notin I R(S, d)$. Because $F$ is Pareto optimal and $S$ is strictly comprehensive, there exists an admissible $T \subset S$ in $\Sigma$ such that $I R(T-d, \mathbf{0})=$ $\operatorname{IR}(S-d, \mathbf{0})$ and $F(S, d) \notin T$. This set $T$ can be obtained by slightly shrinking the non-individually rational part of $S$. IR-invariance requires $F(T, d)=$ $F(S, d)$, which is impossible because $F(S, d) \notin T$.

The next theorem shows that, in combination with maximin efficiency, IRinvariance can be used to characterize the monotone path solutions. This characterization differs from those that can be found in the earlier literature in that it is largely based on a disagreement point axiom - changes in the feasible set only enter through the IR-invariance axiom.

Theorem 3. A solution $F$ is maximin efficient and IR-invariant if, and only if, it is a monotone path solution.

Proof. The if-part is obvious. For the only-if part let $F$ be a solution satisfying maximin efficiency and IR-invariance. By Theorem 2 and Lemma 2, $F$ is an individually rational generalized monotone path solution. We have to show that all the paths involved in generating this solution are identical.

As a first step, consider two feasible sets $V, W \in \Sigma$ whose boundaries $P(V)$ and $P(W)$ are hyperplanes. Let $\Phi^{V}$ and $\Phi^{W}$ denote the corresponding monotone paths according to $F$, and take an arbitrary real number $t>0$ and points $v \in I(V)$ and $w \in I(W)$ such that $F(V, v)-v$ is the point of $\Phi^{V}$ with sum of the coordinates equal to $t$, and $F(W, w)-w$ is the point of $\Phi^{W}$ with sum of the coordinates equal to $t$. Next, let $d, d^{\prime} \in I(V \cap W)$ be such that $I R(V \cap W-d, \mathbf{0})=I R(V-v, \mathbf{0})$ and $I R\left(V \cap W-d^{\prime}, \mathbf{0}\right)=I R(W-w, \mathbf{0})$. $I R$-invariance applied twice then yields that, up to the point with sum of the coordinates equal to $t$, the monotone paths $\Phi^{V}$ and $\Phi^{W}$ must coincide because they both coincide with $\Phi^{V \cap W}$ up to $t$. Since $t$ was chosen arbitrarily, we conclude that $\Phi^{V}=\Phi^{W}$. Therefore, the monotone paths corresponding to the values of the solution $F$ for admissible sets determined by hyperplanes are identical. See Fig. 3 for an illustration of this construction.

Finally, let $(S, d) \in \mathscr{B}$ be arbitrary. In view of the preceding step, it is sufficient to prove that $\{F(S, d)\}=\left(d+\Phi^{V}\right) \cap P(S)$ for some admissible set $V \in \Sigma$ determined by an arbitrary hyperplane. Let $t>0$ be equal to the sum of the coordinates of $F(S, d)-d$. By the strict comprehensiveness of $S$, it is possible to find such a set $V \in \Sigma$ and a disagreement point $v \in I(V \cap S)$ such that $I R(S-d, \mathbf{0})=I R(S \cap V-d, \mathbf{0}), I R(V-v, \mathbf{0})=I R(S \cap V-v, \mathbf{0})$, and $F(V, v)-v$ has sum of the coordinates equal to $t-$ see Fig. 4 . The desired result now follows by applying $I R$-invariance twice.

Unlike earlier axiomatizations of monotone path solutions, the above results are based on disagreement point axioms rather than axioms using varia- 


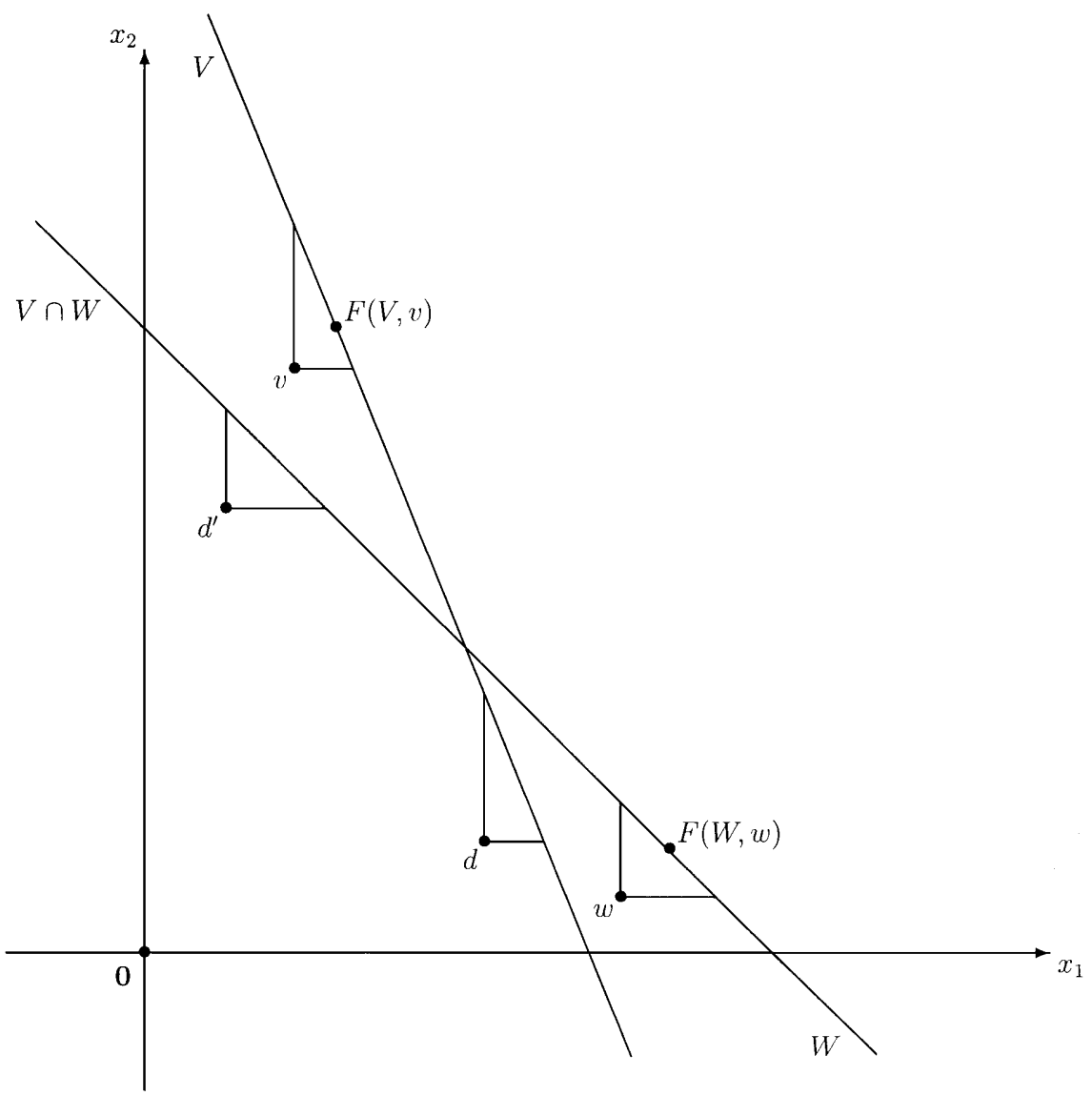

Fig. 3 Proof of Theorem 3, first step

tions in the feasible set. The domination axiom implied by maximin efficiency bears some conceptual resemblance to Kalai's (1977) strong monotonicity axiom which imposes a dominance relationship between the solution outcomes for a feasible set and a subset thereof.

\section{Alternative decision criteria}

As is the case for solutions to bargaining problems under uncertainty regarding the feasible set (see Bossert et al. 1996), applying the maximax decision criterion leads to rather undesirable conclusions. In particular, if efficiency with respect to the maximin criterion is replaced with maximax efficiency, we obtain an impossibility result.

Theorem 4. There exists no maximax efficient solution. 


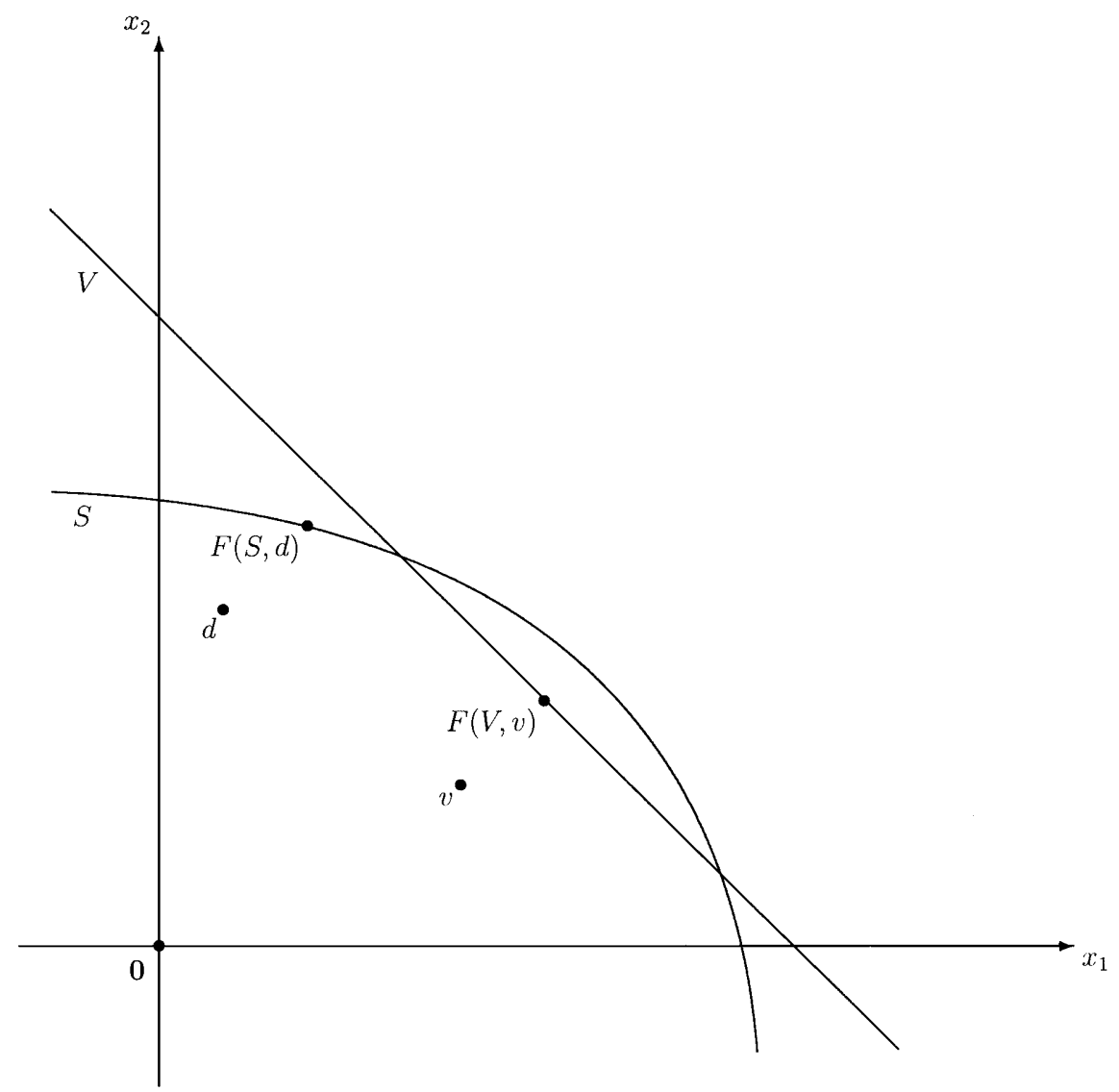

Fig. 4 Proof of Theorem 3, second step

Proof. Let $F$ be a solution, and consider any $(S, d) \in \mathscr{B}$ and $i, j \in N$ with $i \neq j$. Because $S$ is strictly comprehensive, there exist $x, x^{\prime} \in S$ such that $x_{i}<F_{i}(S, d)$, $x_{k}>F_{k}(S, d)$ for all $k \in N \backslash\{i\}, \quad x_{j}^{\prime}<F_{j}(S, d)$, and $x_{k}^{\prime}>F_{k}(S, d)$ for all $k \in N \backslash\{j\}$. Then $\left(x, x^{\prime}\right)$ max-dominates $(F(S, d), F(S, d))$, which proves that $F$ cannot be maximax efficient.

In order to avoid this impossibility result, the above maximax efficiency requirement could be weakened by requiring $x$ and $x^{\prime}$ to be in the individually rational portion of $(S, d)$ and $\left(S, d^{\prime}\right)$, respectively. In that case, dictatorial solutions (among others) become available.

Another alternative to the maximin criterion is to use minimax regret. As in Bossert and Peters (2001), this leads, in the individually rational case, to solutions that are dual to those described in Theorems 2 and 3, where monotone paths originating from the utopia point (the vector of maximal payoffs of the agents within the individually rational portion of a problem) rather than the disagreement point are used. Of course, these solutions are not well- 
defined without individual rationality because utopia points do not exist in that case. See Bossert and Peters (2001) for a formal definition and a discussion of minimax regret in the context of bargaining problems with uncertain feasible sets.

Finally, we note that the expected utility criterion leads to rather degenerate solutions. Expected utility efficiency can be defined analogously to maximin and maximax efficiency by employing the expected utility criterion. That is, for all $S \in \Sigma$, for all $d, d^{\prime} \in I(S)$, and for all $\pi \in(0,1)$, there exist no $x, x^{\prime} \in S$ such that

$$
\pi(x-d)+(1-\pi)\left(x^{\prime}-d^{\prime}\right)>\pi(F(S, d)-d)+(1-\pi)\left(F\left(S, d^{\prime}\right)-d^{\prime}\right) .
$$

Note that this inequality is equivalent to

$$
\pi x+(1-\pi) x^{\prime}>\pi F(S, d)+(1-\pi) F\left(S, d^{\prime}\right) .
$$

Consider any $S \in \Sigma$ such that $S$ is strictly convex. Suppose there exist $d, d^{\prime} \in I(S)$ such that $F(S, d) \neq F\left(S, d^{\prime}\right)$. Because $S$ is strictly convex, there exists $x \in S$ such that $x>\pi F(S, d)+(1-\pi) F\left(S, d^{\prime}\right)$. Letting $x^{\prime}=x$, we obtain the above inequality and, thus, a contradiction to expected utility efficiency. Therefore, there must exist a constant vector $c \in P(S)$ such that $F(S, d)=c$ for all $d \in I(S)$ and hence, the solution is constant in $d$ given any strictly convex $S$. As is the case for maximax efficiency, further solutions become available if expected utility efficiency is weakened by requiring $x$ and $x^{\prime}$ to be in the individually rational portion of the relevant bargaining problem. As can be checked by an argument similar to the foregoing, these solutions still are insensitive with respect to changes in $d$ over large regions of $I(S)$ for strictly convex $S$. These observations suggest that expected utility efficiency does not lead to solutions of much interest.

\section{References}

Arrow KJ, Hurwicz L (1972) An optimality criterion for decision-making under ignorance. In: Carter CF, Ford JL (eds) Uncertainty and expectations in economics: Essays in honour of G.L.S. Shackle. Basil Blackwell, Clifton, NJ, pp 1-11

Barberà S, Barrett CR, Pattanaik PK (1984) On some axioms for ranking sets of alternatives. J Econ Theory 33: 301-308

Bossert W (1994) Disagreement point monotonicity, transfer responsiveness, and the egalitarian bargaining solution. Soc Choice Welfare 11: 381-392

Bossert W (1997) Uncertainty aversion in nonprobabilistic decision models. Math Soc Sci 34: 191-203

Bossert W, Nosal E, Sadanand V (1996) Bargaining under uncertainty and the monotone path solutions. Games Econ Behav 14: 173-189

Bossert W, Pattanaik PK, Xu Y (2000) Choice under complete uncertainty: axiomatic characterizations of some decision rules. Econ Theory 16: 295-312

Bossert W, Peters H (2001) Minimax regret and efficient bargaining under uncertainty. Games Econ Behav 34: 1-10

Chun Y (1989) Lexicographic egalitarian solution and uncertainty in the disagreement point. Z Oper Res 33: 259-306 
Chun Y, Thomson W (1990a) Bargaining with uncertain disagreement points. Econometrica 58: 951-959

Chun Y, Thomson W (1990b) Egalitarian solutions and uncertain disagreement points. Econ Lett 33: 29-33

Chun Y, Thomson W (1990c) Nash solution and uncertain disagreement points. Games Econ Behav 2: 213-223

Gilboa I, Schmeidler D (1989) Maxmin expected utility with a non-unique prior. J Math Econ 18: 141-153

Kalai E (1977) Proportional solutions to bargaining situations: interpersonal utility comparisons. Econometrica 45: 1623-1630

Kalai E, Smorodinsky M (1975) Other solutions to Nash's bargaining problem. Econometrica 43: 513-518

Kannai Y, Peleg B (1984) A note on the extension of an order on a set to the power set. J Econ Theory 32: 172-175

Livne Z (1988) The bargaining problem with an uncertain conflict outcome. Math Soc Sci 15: 287-302

Livne Z (1989) On the status quo sets induced by the Raiffa and the KalaiSmorodinsky solutions to the bargaining problem. Math Oper Res 14: 688-692

Nash J (1950) The bargaining problem. Econometrica 18: 155-162

Pattanaik PK, Peleg B (1984) An axiomatic characterization of the lexicographic maximin extension of an ordering over a set to the power set. Soc Choice Welfare 1: $113-122$

Peters H (1992) Axiomatic Bargaining Game Theory. Kluwer, Dordrecht, The Netherlands

Peters H, van Damme E (1991) Characterizing the Nash and Raiffa solutions by disagreement point axioms. Math Oper Res 16: 447-461

Thomson W (1987) Monotonicity of bargaining solutions with respect to the disagreement point. J Econ Theory 42: 50-58

Thomson W (1994) Cooperative models of bargaining. In: Aumann R, Hart S (eds) Handbook of game theory, vol. 2. Elsevier, Amsterdam, The Netherlands, pp 1237-1284

Thomson W, Myerson R (1980) Monotonicity and independence axioms. Int J Game Theory 9: 37-49

Wakker P (1987) The existence of utility functions in the Nash solution for bargaining. In: Paelinck JHP, Vossen PH (eds) Axiomatics and pragmatics of conflict analysis. Gower, Aldershot, UK, pp 157-177

Wald A (1950) Statistical Decision Functions. Wiley, New York 